\title{
Effects of thermal cycling parameters on residual stresses in alumina scales of CoNiCrAlY and NiCoCrAIY bond coats
}

\author{
Christian Nordhorn ${ }^{\text {a }}$, Robert Mücke ${ }^{\mathrm{a}}$, Kinga A. Unocic ${ }^{\mathrm{b}}$, Michael J. Lance ${ }^{\mathrm{b}}$, \\ Bruce A. Pint ${ }^{\mathrm{b}}$, Robert Vaßen ${ }^{\mathrm{a}}$
}

${ }^{a}$ IEK-1, Forschungszentrum Jülich GmbH, Wilhelm-Johnen-Straße, 52428 Jülich, Germany
${ }^{\mathrm{b}}$ Materials Science and Technology Division, Oak Ridge National Laboratory, Oak Ridge, TN 37831, United States

Furnace cycling experiments were performed on free-standing high-velocity oxygen-fuel bond coat samples to investigate the effect of material composition, surface texture, and cycling conditions on the average stresses in the formed oxide scales after cooling. The oxide scale thicknesses were determined by SEM image analyses and information about the stresses were acquired by photo-stimulated luminescence-spectroscopy. Additionally, the scale thickness dependent stress fields were calculated in finite-element analyses including approximation functions for the surface roughness derived on the basis of profilometry data. The evolution of the average residual stress as a function of oxide scale thickness was subject to stochastic fluctuations predominantly caused by local scale spallations. In comparison to the supplementing modeling results, thermal stresses due to mismatch of thermal expansion coefficients are identified as the main contribution to the residual stresses. The theoretical results emphasize that analyses of spectroscopic data acquired for average stress investigations of alumina scales rely on detailed information about microstructural features.

\footnotetext{
${ }^{1}$ Corresponding author. Tel.: +49 2461 615357. E-mail address: c.nordhorn@fz-juelich.de
} 
Keywords: Photo-stimulated luminescence-spectroscopy, Thermally grown oxide, MCrAlY bond coats, Finite-element analysis, Mechanical stress 


\section{Introduction}

The application of plasma sprayed thermal barrier coating (TBC) systems on heat exposed components of gas turbines is an established procedure to both increase the energy conversion efficiency and enhance the turbine durability. The protective coating systems consist typically of two functional layers, a ceramic topcoat providing the thermal resistivity and a metallic bond coat $(\mathrm{BC})[1,2]$. A pronounced $\mathrm{BC}$ surface roughness facilitates the bonding of the topcoat to the substrate, which is additionally protected by the $\mathrm{BC}$ layer from detrimental oxidation. During operation, a thermally grown oxide (TGO) layer is formed at the interface between the $\mathrm{BC}$ and the topcoat. Failure of the topcoat, which is equivalent to lifetime expiration of the complete TBC system, is correlated to the oxide scale growth due to its impact on the thermally induced stress fields, which promote crack propagation as a consequence of cyclic loading [3, 4]. Because of the relatively high content of aluminum in common $\mathrm{BC}$ alloy compositions, the TGO layer is predominantly composed of $\alpha-\mathrm{Al}_{2} \mathrm{O}_{3}$. The presence of $\mathrm{Cr}^{3+}$ substitution ions in these $\alpha$-alumina scales grants experimental access to monitoring stress evolution in the growing scales. In a non-destructive way, photo-stimulated luminescence-spectroscopy (PSLS) allows the investigation of stress induced peak shifts of luminescence spectra from the $\mathrm{Cr}^{3+}$ substitution ions, which can provide information about the integrity of the TBC system to assess its remaining lifetime on an empirical basis [5-7]. However, in general, these empirical insights cannot be transferred non-restrictively to lifetime analyses of TBC systems applied on turbine components, because several advanced problems like the geometric specifications have to be considered [8]. Moreover, under real operating conditions contamination of the topcoat by exhaust-gas impurities can perturb investigations by PSLS because of falsification of the spectra or blocking of the analysis light pathways due to the optical properties of the contaminants [9].

The PSLS data can also be used indirectly to draw conclusions concerning the durability of the coating systems, instead of monitoring the stress development directly. The alternative, 
indirect approach for processing the information about the stress state is to compare the experimental results to corresponding finite-element (FE) analyses, which have their basic principles in common with FE simulations implemented in TBC lifetime models [10-13]. An agreement between experiment and theory indicates principal model capability regarding the stress level determinations and the results of this comparison serve as feedback about the significance of the FE analysis. On the basis of verified lifetime models, predictions concerning input parameter variations are possible.

To explore this strategy, thermal cycling experiments were performed utilizing free-standing $\mathrm{BC}$ samples in order to measure the thicknesses of the formed oxide scales and the average residual stresses. These results were obtained for several sets of samples comprising two different $\mathrm{BC}$ materials with two different surface textures from furnace cycling experiments under six different cycling conditions. The comprehensive input parameter space of the study enabled the analysis of the effect of a multitude of parameters including the addition of watervapor during thermal cycling, which can have detrimental effects on TBC performance [14, $15]$.

The experiments were complemented by FE analyses of the stress distributions in the TGO scales. To facilitate a comparison to two-dimensional models used in lifetime simulations and a check of the implemented boundary conditions, the material parameters, and the approximation of the surface roughness, two-dimensional FE models were also used to simulate the thermally induced stress fields of the BC-TGO systems. If accordance to experimental results is achieved, the individual mentioned FE model features will be confirmed within the scope of this study giving some justification for the use of these features in lifetime models. 


\section{Experiments}

Two large-scale $\mathrm{BC}$ coatings $(100 \mathrm{~mm} \times 50 \mathrm{~mm}$ ) were produced by high-velocity oxygen-fuel (HVOF) thermal spraying on steel substrates using a DJ2600 gun (Sulzer Metco, Wohlen, Switzerland) with a robot speed of $500 \mathrm{~mm} / \mathrm{s}$ and a spray distance of $200 \mathrm{~mm}$ [16]. The nominal compositions of the used thermal spray powders, one Co-based and one Ni-based powder, are shown in Table I. The coatings were sprayed at an oxygen-fuel/hydrogen ratio with flows of 165/630 standard liters per minute (SLPM) for the Co-based and 155/630 SLPM for the Ni-based powder. The shroud nitrogen flows were 460 SLPM and 430 SLPM, respectively. After separation from the steel substrates by electrical discharge machining $(\mathrm{EDM})$ the free-standing large-scale samples were cut into smaller samples with dimensions of $10 \mathrm{~mm} \times 10 \mathrm{~mm} \times 2 \mathrm{~mm}$. The experimental setup required the machining of holes in the samples, located approximately $2 \mathrm{~mm}$ from one of the edges, with a diameter of about $1 \mathrm{~mm}$. These holes were either drilled or, due to a more efficient preparation, machined by EDM (referred to as EDM samples) as shown in Table II. Residue from the EDM process was ground off except for inside the hole. Additionally, the two $100 \mathrm{~mm}^{2}$ faces of each sample were ground by 120 grit and 600 grit SiC-paper, respectively, in order to create two different isotropic surface textures on the opposing surfaces of each specimen. The resulting surface roughness parameters were determined by profilometry (CT350T with CHR1000 chromatic sensor, cyberTECHNOLOGIES GmbH, Ingolstadt, Germany). The mean arithmetic average roughness, $\mathrm{S}_{\mathrm{a}}$, and the root mean square slope, $\mathrm{S}_{\mathrm{dq}}$, are displayed in Table III. Furnace cycling experiments were conducted in automated cyclic rigs [17] where four Cobased and four Ni-based free-standing BC samples were cycled together. One cycle comprised one hour at high temperature in the furnace and ten minutes cooling in air after retraction of the samples from the furnace. The temperature and environment of the different experiments are shown in Table II. One Co-based and one Ni-based BC sample were removed from the cycling experiments after 5, 20, 40, and 100 cycles, respectively. Consequently, 
there is one sample surface for each possible combination of the five parameters sample material, surface texture, cycling temperature, cycling environment, and cycling duration. After thermal cycling, the oxide scales were investigated regarding their average residual stress state at room temperature by PSLS and regarding their thickness by image analyses based on SEM images of sample cross-sections. The high $\alpha$-alumina content of the TGO scales enabled measurement of the residual stresses by spectrometry utilizing the piezospectroscopic effect. The luminescence signal of the $\mathrm{Cr}^{3+}$ substitution ions in the $\mathrm{Al}_{2} \mathrm{O}_{3}$ scale depends on the stress state [18]. The PSLS was performed using the experimental setup outlined previously [19]. A detailed description of the experimental concept is also given in [20]. Therefore, only the main setup characteristics will be explained briefly at this point. An argon-ion laser (Innova 308c, Coherent, Inc., Santa Clara, USA) operating at a wavelength of $514.5 \mathrm{~nm}$ was used to excite the chromium ions in the alumina scale. The luminescence spectra were collected with a Raman microprobe (Dilor XY800, JY, Inc., Edison, USA). Eleven measurements with five seconds acquisition time along a $5 \mathrm{~mm}$ line scan were performed at room temperature. The diameter of the defocused excitation beam on the sample surface was set to approximately $500 \mu \mathrm{m}$, so that a multitude of grains was excited and not individual grains. The responding luminescence signals of all chromium ions, distributed over the entire scale thickness, were collected, because no other ions respond to the excitation beam [21]. The measurements were performed at low laser power to prevent heating of the sample.

The peak position $v$ of the $\mathrm{R} 2$ peak, which is linearly correlated to the stress state of the corresponding crystals [18], is extracted from the luminescence signal by a pseudo-Voigtian (weighed sum of a Gaussian and a Lorentzian) double peak fit, which reflects the emissionspectrum features of ruby in this wavelength-range, including a baseline for the $\theta$-alumina contribution [22] to the signal and compared to the reference position. The measurement of this reference peak position $v_{0}$ was performed with the same setup using alumina powder 
(NIST standard reference material 676a), in which the substitution chromium ions are considered as being stress-free. Fig. 1 shows an example of a measured luminescence spectrum of an oxide scale under stress and its pseudo-Voigtian fit in comparison to the alumina-powder reference spectrum. According to [18], the stress $(\sigma)$ induced shift $\Delta v=v-$ $v_{0}$ of the R2 peak depends on the piezospectroscopic tensor $\Pi$ and is generally given by Eq. (1).

$$
\Delta v=\Pi_{i j} \sigma_{i j}
$$

The piezospectroscopic coefficients for the R2-line of ruby are given in [23]. Given a scale growth with the alumina c-axis being oriented perpendicular to the interface, Eq. (1) can be restated resulting in Eq. (2) for the biaxial stress $\sigma$.

$$
\sigma=\frac{\Delta v}{\Pi_{11}+\Pi_{22}}
$$

Assuming a polycrystalline material with randomly oriented grains under homogeneous stress, the average peak shift $\overline{\Delta v}$ for ruby results from Eq. (3) [18].

$$
\overline{\Delta v}=\frac{1}{3} \Pi_{i i} \sigma_{i j}
$$

Due to the low roughness values, the normal stress component perpendicular to the interface is decisively smaller than the in-plane components. Consequently, the mean biaxial stress $\bar{\sigma}$ is given by Eq. (4).

$$
\bar{\sigma}=\frac{3}{2} \frac{\overline{\Delta v}}{\Pi_{i i}}
$$

The TGO scale thicknesses were determined by image analyses using ImageJ software (National Institutes of Health, Bethesda, USA) based on SEM cross section images. The SEM images were taken in Oak Ridge using a Hitachi S4800 (Hitachi Europe GmbH, Düsseldorf, 
Germany) and with a Zeiss Gemini Ultra 55 (Carl Zeiss NTS GmbH, Oberkochen, Germany) in Jülich. Eight images were taken for each surface. The thickness of the TGO layer normal to the BC interface was determined at eight predefined equidistant positions on all eight SEM images for each sample, independently of the composition of the TGO scale. To ensure comparability of the SEM images from two different microscopes, four surface cross-sections were analyzed based on images from both microscopes. The results of these measurements did not show significant deviations. 


\section{Calculations}

The thermally induced stresses in the TGO scales were calculated under the assumption of a stress-free high-temperature state. This assumption is based on the creep rates of the involved materials, which are sufficiently high [24] for complete stress relaxation, independent of the cycling conditions within the scope of the chosen parameter space.

A two-dimensional FE model was implemented using ANSYS, version 14.5 (ANSYS, Inc., Canonsburg, USA). It comprised half of the BC sample height and the TGO layer with variable height $d_{T G O}$. The mesh was constructed, so that TGO growth perpendicular to the original interface can be simulated in steps of $\Delta d_{T G O}=0.1 \mu \mathrm{m}$. The textures of the two differently ground sample surfaces were taken into account by a cosine function. The corresponding amplitude $A$ and wavelength $L$ of this approximation function were derived on the basis of an equation system including the formulas for the arithmetic average roughness $S_{a}$ , Eq. (5), and the root mean square slope $S_{d q}$, Eq. (6) as parametric functions of a twodimensional surface generated by two cosine functions, Eq. (7).

$$
\begin{gathered}
S_{a}=\frac{1}{M N} \sum_{k=1}^{M} \sum_{l=1}^{N}\left|z\left(x_{k}, y_{l}\right)\right| \\
S_{d q}=\sqrt{\frac{1}{(M-1)(N-1)} \sum_{k=2}^{M} \sum_{l=2}^{N}\left(\frac{z\left(x_{k}, y_{l}\right)-z\left(x_{k-1}, y_{l}\right)}{\Delta x}\right)^{2}+\left(\frac{z\left(x_{k}, y_{l}\right)-z\left(x_{k}, y_{l-1}\right)}{\Delta y}\right)^{2}} \\
z(x, y)=A \cos \left(\frac{2 \pi}{L} x\right) \cos \left(\frac{2 \pi}{L} y\right)
\end{gathered}
$$

The solutions of this equation system and the measured surface roughness parameters, which served as input parameters, are displayed in Table III.

Within the FE model, the TGO was assumed to consist completely of $\alpha-\mathrm{Al}_{2} \mathrm{O}_{3}$. The stress calculations required the Young's modulus and the Poisson's ratio at room temperature, which were chosen as $E_{T G O}=400 \mathrm{GPa}$ and $v_{T G O}=0.23$, respectively [21]. The corresponding BC parameters were $E_{B C}=220 \mathrm{GPa}$ and $v_{B C}=0.3$. The coefficients of thermal expansion 
were defined as a function of temperature and were calculated based on literature data for the BC materials [25] and for $\alpha$-alumina [26]. The calculated data are displayed in Table IV. The thermally induced stresses were calculated by applying isothermal loads. The lower boundary of the model was kept fixed. Periodic boundary-conditions were defined by constraint equations at the lateral nodes. The strain perpendicular to the model plane was defined by employing the generalized plane strain feature, which enabled the definition of a deformation domain in the virtual third model dimension, under the assumption that the stresses in the normal directions parallel to the interfaces were of equal values. 


\section{Results}

The average thicknesses (of 64 measurements) of the TGO layers are displayed in Fig. 2 as a function of the high-temperature exposure time. The values refer to the entire TGO scale independently of its composition. The corresponding standard errors are generally smaller than the symbol size and represented by the largest error obtained for the rougher side of the Co-based samples (Fig. 2 a). For clarity reasons, the data are presented in separate diagrams subdivided according to material composition and surface texture. Each sample surface contributes to this figure with exactly one data point. Consequently the time-dependent evolution cannot be assigned to one TGO layer growth, but rather four independently grown scales. Non-monotonous behavior of the curves can be attributed to this methodology. The samples cycled at $950^{\circ} \mathrm{C}$ were not investigated by SEM and subsequent image analyses, because the scale was expected to be too thin, based on low PSLS signal intensities. Representative SEM images of the smoother interface of the Co-based and the rougher interface of the Ni-based sample, which were cycled at $1100^{\circ} \mathrm{C}$ for $20 \mathrm{~h}$, are shown in Fig. 3 . The EDM samples exposed at $1000^{\circ} \mathrm{C}$ showed higher oxide thicknesses than expected (Fig. 2) presumably because of contamination during the EDM process.

The cycling atmosphere had no significant influence on the TGO thicknesses of the samples cycled at $1050^{\circ} \mathrm{C}$, which were not made by EDM. Comparing specimens individually, whose cycling parameters only differ with respect to the atmosphere composition, the TGO thickness was slightly but not substantially higher after cycling with the addition of water vapor, typically. An effect of the surface texture could not be resolved. For the Co-based samples, a faster TGO growth was detected compared to the Ni-based samples.

The results of the PSLS measurements converted to the mean biaxial stress according to Eq. (4) are shown in Fig. 4, again divided by the two BC compositions and surface roughnesses. The average residual stresses in the TGO scales after cooling and the corresponding standard errors are shown as a function of the measured scale thicknesses. The TGO scales are all 
under compressive stress after cooling. As a general trend, these compressive stresses either increase slightly or remain constant with increasing scale thickness. Exceptions to this trend are the Co-based samples with rougher surface texture (Fig. 4 a) and the Ni-based samples with the lower surface roughness (Fig. 4 d), both cycled at $1050^{\circ} \mathrm{C}$. As expected, the compressive stresses were higher for samples cycled at higher temperatures. The effect of atmosphere on the measured stress was ambiguous. For the majority of the samples cycled at $1050^{\circ} \mathrm{C}$, the addition of water vapor led to higher compressive stresses compared to furnace cycling in dry air. However, the data collected for the 600 grit surfaces of the Co-based samples (Fig. 4 b), do not support this conclusion, but rather reveal no significant atmosphere effect. After cycling at $1000^{\circ} \mathrm{C}$ in wet air, the stresses on the 120 grit surfaces were lower for the Co-based samples (Fig. 4 a) and higher for the Ni-based samples (Fig. 4 c). Because of the strongly scattered data in the other two diagrams no clear conclusions were possible in these cases. In general, the stresses measured in the TGO scales formed on Ni-based samples were higher than those measured in the TGO scales formed on the Co-based BC samples. On the smoother surfaces, the stresses were slightly higher than on the rougher, 120 grit surfaces. In a direct comparison between the rougher and the smoother surface of individual samples, the stresses on the 600 grit sides were higher for approximately $75 \%$ of the samples.

Fig. 4 also shows a comparison between the calculation results and the measured stresses. The theoretical results are represented by the patterned areas, whose center lines are given by the mean normal stresses in the $x$-direction. For the highest cycling temperature, an additional error bar takes an alternative evaluation of the peak shifts given by Eq. (2) into account, which considers columnar TGO growth and will be explained in the following section. The calculated mean stress was virtually independent of the TGO thickness within the scope of the FE modeling results. For the rougher surface of the Ni-based samples (Fig. 4 c) for example, the average compressive stress amounts to approximately $3.28 \mathrm{GPa}$ for cycling at $1100^{\circ} \mathrm{C}$, $3.13 \mathrm{GPa}$ for cycling at $1050^{\circ} \mathrm{C}$, and $2.98 \mathrm{GPa}$ for cycling at $1000^{\circ} \mathrm{C}$. However, the 
corresponding standard deviations, which were utilized as a measure for the heterogeneity of the stress fields in the TGO, increased with the growing oxide scale. Fig. 5 shows the stress field distribution of the normal stress in the $x$-direction exemplarily for a $3 \mu \mathrm{m}$ thick TGO scale on the rougher surface of a Ni-based sample cycled at $1000^{\circ} \mathrm{C}$. For thinner scales, the structure of the stress distribution was maintained, but the spectrum of occurring stresses was smaller. On the smoother surfaces the simulated stresses were slightly higher and showed less variance, which can be attributed to the smaller roughness values. 


\section{Discussion}

The mass change data are not shown because they were not required for the analyses of the residual stress as a function of oxide scale thickness.

In general, the PSLS experiments yielded, as intermediate result, the mean peak shifts, derived from the luminescence spectra by a pseudo-Voigtian fit, which did not take possible substructures of the spectra into account. These substructures could principally be assigned to different peak shifts indicating multiple different stress levels, which are detectable by applying stress mapping techniques with higher spatial resolution [19]. Local spallation of the TGO scales led to a reduction of the biaxial stress levels, influencing the measurements of the average peak shifts. At the same time, such localized spallation exposed the BC surface initiating further TGO growth. The uncertainty about the extent to which spallation and subsequent regrowth proceed implies uncertainty concerning determination of the average stress levels in the TGO scale. The longer a sample is cycled, the higher is the probability for spallation. This correlation is indicated by the relatively large errors obtained for the stresses in the TGO scales on the Co-based specimens in Fig. 4.

The effect of water vapor addition on the residual stress levels is ambiguous because of different oxide growth kinetics, which influences multiple opposing effects. The presence of water vapor during thermal cycling leads to an increase of the oxidation rate of HVOF NiCoCrAlY BCs and promotes the formation of spinel phases [27]. Moreover, the rate of scale spallation from bare BC surfaces is increased [14]. This accelerated oxide growth and the enhanced spallation probability introduce further uncertainty factors adding to the randomly distributed incidences of scale spallation and subsequent regrowth, which have opposite impacts on the average stress in the scale.

Independent of the cycling atmosphere, the stress evolution as a function of TGO scale thickness is subject to these stochastic processes and consequently shows no clear trend in the corresponding figures. 
In most cases, the stresses detected on the smoother surface of an individual sample were higher than the stresses on the opposite, rougher side. The smaller geometric surface gradient of the 600 grit surfaces prevents a redirection of the predominant in-plane stresses into the vertical component. Therefore, the average in-plane stresses were higher.

The accompanying theoretical evaluations focused on the thermally induced stresses within the TGO scales. Possible additional influences, which were not considered in the calculations, arise from (1) phase transformations and associated changes in the coefficients of thermal expansion [28], which are possible due to the fact that the BC samples were not heat-treated before thermal cycling, and (2) oxidation related volumetric expansion. The accuracy of the modeling results is directly linked to the accuracy of the input parameters, especially the information about the material properties. Moreover, the FE model utilized an approximation function for the surface texture, which did not reflect possible local deviations, because the surface structure was abstracted based on the surface roughness parameters. Considering this, the variance of the calculated average stresses is underestimated owing to the simplified geometry, because calculations based on a deviating approximation function would result in different stress distributions.

The model correctly predicted the slightly higher stresses observed on smoother specimen surfaces as well as, qualitatively, the effect of temperature. However, the relative position of the single evolutions for the three cycling temperatures shows deviations. For the two lower temperatures shown in Fig. 4, the relative position of the experimental values is reflected by the shift of the theoretical results for $1000^{\circ} \mathrm{C}$ against the results for the next higher temperature. The experimental data obtained for the highest cycling temperature were exposed. The mean biaxial stresses were calculated based on Eq. (4) assuming a uniform distribution of the crystallographic axes. This assumption does not necessarily apply to the specimens cycled at $1100^{\circ} \mathrm{C}$. The TGO scales of these specimens are characterized by a columnar microstructure $[29,30]$, in which the c-axis of the alumina crystal system is oriented 
preferentially parallel to the growth direction $[31,32]$. Similar microstructure related results were not found for the other two cycling temperatures. For the data set cycled at $1100^{\circ} \mathrm{C}$, the corresponding conversion was additionally performed using Eq. (2), with the applied stress being imposed on the crystal along its crystallographic axes, and considered in Fig. 4 by an additional error bar. This second error bar gives an estimation of a systematic error, which can be attributed to the conversion of the spectrometry data. It takes into account that the stresses derived on the basis of the measured peak-shifts may be overestimated by using Eq. (4) for the evaluation. Considering this additional shift, the relative differences between the single stress evolutions are described by the relative positions of the theoretical results more exactly. The major contribution to stresses appears to be caused by the mismatch of the thermal expansion coefficients.

The effect of increased oxidation rates of the EDM samples is not well understood. Presumably, the slightly porous BC samples (3.4\% average porosity determined by image analysis) were contaminated by impurities of the dielectric fluid, which was employed during machining. The presence of these contaminants might have potentially led to the enhanced oxide growth on the affected BC samples. The contaminated fluid would have been loosely attached to the free-standing BC sample and unaffected by subsequent thermal cycling preparations. To prove this hypothesis, one Co- and one Ni-based EDM sample were annealed in a vacuum for $10 \mathrm{~h}$ at $1000^{\circ} \mathrm{C}$ before thermal cycling in air at the same temperature. After 20 cycles, these samples showed mass gains, which were consistent with the first set of samples without EDM holes.

An influence of the presumed impurities on the PSLS results is not expected, because the luminescence spectra of exclusively the $\mathrm{Cr}^{3+}$ ions in the formed alumina scales were recorded. The stress results were plotted versus the TGO scale thickness, so that the enhanced scale growth for the $\mathrm{BC}$ samples cycled at $1000^{\circ} \mathrm{C}$ was included in the data presentation of the stress measurements. 


\section{Conclusions}

The residual stresses in alumina scales formed on CoNiCrAlY and NiCoCrAlY BCs were measured by PSLS in order to detect possible effects of several parameters varied in thermal cycling experiments. The effects of exposure temperature and water vapor addition were clearly noticeable. However, the latter was ambiguous due to uncertainties arising from data evaluation based on average stress values. Moreover, average stress analyses by PSLS require detailed information about the microstructure of the investigated scale, which determines how the directly measured peak shifts are converted into stresses. Regarding monitoring of stress evolution, stress maps turn out to be more informative because of the essentially higher spatial resolution. Considerable local deviations in the stress distributions, which are attributed to spallations and possible subsequent regrowth amongst others, are not detectable by averaging, but can be identified by the mapping technique.

The comparison of the experimental results to the results of FE analyses, which were adapted to the given experimental conditions, yielded qualitatively consistent findings with regard to the temperature and the surface texture effect. The major contribution to the experimentally detected stresses are thermal stresses. Moreover, the stress states are dependent on stress reduction as a consequence of spallation and oxidation induced stresses.

The implementation of the boundary conditions and the definition of the material parameters led to results within the scope of the FE modeling, which are in general agreement with the measured data. In this respect, their application in more comprehensive FE models dealing with thermo-mechanical stress analyses of related systems was substantiated. 


\section{Acknowledgments}

The authors gratefully acknowledge the support of D. Sebold for SEM work at Jülich, G.W. Garner at ORNL for assistance with the thermal cycling experiments, and T.M. Lowe and T. Jordan for assistance with the experimental work at ORNL. The experimental work at ORNL was supported by U.S. Department of Energy, Office of Coal and Power R\&D, Office of Fossil Energy. 


\section{References}

[1] A.G. Evans, D.R. Mumm, J.W. Hutchinson, G.H. Meier, F.S. Pettit, Mechanisms controlling the durability of thermal barrier coatings, Progr. Mater. Sci. 46 (2001) 505-553

[2] N.P. Padture, M. Gell, E.H. Jordan, Thermal Barrier Coatings for Gas-Turbine Engine Applications, Science 296 (2002) 280-284

[3] A.G. Evans, M.Y. He, J.W. Hutchinson, Mechanics-based scaling laws for the durability of thermal barrier coatings, Progr. Mater. Sci. 46 (2001) 249-271

[4] J. Schwarzer, D. Löhe, O. Vöhringer, Influence of the TGO creep behavior on delamination stress development in thermal barrier coating systems, Mater. Sci. Eng. A 387389 (2004) 692-695

[5] D.R. Clarke, R.J. Christensen, V. Tolpygo, The evolution of oxidation stresses in zirconia thermal barrier coated superalloy leading to spalling failure, Surf. Coat. Technol. 94-95 (1997) 89-93

[6] J.A. Nychka, D.R. Clarke, Damage quantification in TBCs by photo-stimulated luminescence spectroscopy, Surf. Coat. Technol. 146-147 (2001) 110-116

[7] X. Peng, D.R. Clarke, Piezospectroscopic Analysis of Interface Debonding in Thermal Barrier Coatings, J. Am. Ceram. Soc. 83 [5] (2000) 1165-1170

[8] X. Wang, G. Lee, A. Atkinson, Investigation of TBCs on turbine blades by photoluminescence piezospectroscopy, Acta Mater. 57 (2009) 182-195

[9] M.S. Majewski, C. Kelley, J. Lake, M.W. Renfro, W. Hassan, W. Brindley, E.H. Jordan, Stress measurements via photoluminescence piezospectroscopy on engine run thermal barrier coatings, Surf. Coat. Technol. 206 (2012) 2751-2758

[10] E.P. Busso, L. Wright, H.E. Evans, L.N. McCartney, S.R.J. Saunders, S. Osgerby, J. Nunn, A physics-based life prediction methodology for thermal barrier coating systems, Acta Mater. 55 (2007) 1491-1503

[11] A.M. Karlsson, A.G. Evans, A numerical model for the cyclic instability of thermally grown oxides in thermal barrier systems, Acta Mater. 49 (2001) 1793-1804

[12] R. Vaßen, S. Giesen, D. Stöver, Lifetime of Plasma-Sprayed Thermal Barrier Coatings: Comparison of Numerical and Experimental Results, J. Therm. Spray Technol. 18 (5-6) (2009) 835-845

[13] P. Seiler, M.Bäker, T. Beck, M. Schweda, J. Rösler, FEM simulation of TBC failure in a model system, J. Phys.: Conf. Ser. 240 (2010) 012069

[14] J.A. Haynes, K.A. Unocic, B.A. Pint, Effect of water vapor on the $1100^{\circ} \mathrm{C}$ oxidation behavior of plasma-sprayed TBCs with HVOF NiCoCrAIX bond coatings, Surf. Coat. Technol. 215 (2013) 39-45 
[15] V. Déneux, Y. Cadoret, S. Hervier, D. Monceau, Effect of Water Vapor on the Spallation of Thermal Barrier Coating Systems During Laboratory Cyclic Oxidation Testing, Oxid. Met. 73 (2010) 83-93

[16] B. Rajasekaran, G. Mauer, R. Vaßen, Enhanced Characteristics of HVOF-sprayed MCrAlY Bond Coats for TBC Applications, J. Therm. Spray Technol. 20 (6) (2011) 12091216

[17] B. A. Pint, G. W. Garner, T. W. Lowe, J. A. Haynes, Y. Zhang, Effect of increased water vapor levels on TBC lifetime with Pt-containing bond coatings, Surf. Coat. Technol. 206 (2011) 1566-1570

[18] Q. Ma, D.R. Clarke, Stress Measurement in Single-Crystal and Polycrystalline Ceramics Using Their Optical Fluorescence, J. Am. Ceram. Soc. 76 [6] (1993) 1433-1440

[19] M. J. Lance, K. A. Unocic, J. A. Haynes, B. A. Pint, Effect of water vapor on thermallygrown alumina scales on Pt-modified and simple aluminide bond coatings, Surf. Coat.

Technol. 237 (2013) 2-7

[20] D.M. Lipkin, D.R. Clarke, Measurement of the Stress in Oxide Scales Formed by Oxidation of Alumina-Forming Alloys, Oxid. Met. 45 3/4 (1996) 267-280

[21] D.M. Lipkin, D.R. Clarke, M. Hollatz, M. Bobeth, W. Pompe, Stress development in alumina scales formed upon oxidation of (111) NiAl single crystals, Corros. Sci. 39 (1997) 231-242

[22] Q. Wen, D.M. Lipkin, D.R. Clarke, Luminescence Characterization of ChromiumContaining $\theta$-Alumina, J. Am. Ceram. Soc. 81 [12] (1998) 3345-3348

[23] J. He, D.R. Clarke, Determination of the Piezospectroscopic Coefficients for ChromiumDoped Sapphire, J. Am. Ceram. Soc. 78 [5] (1995) 1347-1353

[24] J. Rösler, M. Bäker, M. Volgmann, Stress state and failure mechanisms of thermal barrier coatings: Role of creep in thermally grown oxide, Acta Mater. 49 (2001) 3659-3670

[25] T.A. Taylor, P.N. Walsh, Thermal expansion of MCrAlY alloys, Surf. Coat. Technol. 177-178 (2004) 24-31

[26] Y.S. Touloukian, R.K. Kirby, R.E. Taylor, Thermal expansion: nonmetallic solids, IFI/Plenum, New York, 1977, p 176

[27] C. Kaplin, M. Brochu, Effects of water vapor on high temperature oxidation of cryomilled NiCoCrAlY coatings in air and low-SO $\mathrm{S}_{2}$ environments, Surf. Coat. Technol. 205 (2011) 4221-4227

[28] J. Toscano, A. Gil, T. Hüttel, E. Wessel, D. Naumenko, L. Singheiser, W.J. Quadakkers, Temperature dependence of phase relationships in different types of MCrAlY-coatings, Surf. Coat. Technol. 202 (2007) 603-607

[29] C.G. Levi, E. Sommer, S.G. Terry, A. Catanoiu, M. Rühle, Alumina Grown during Deposition of Thermal Barrier Coatings on NiCrAlY, J. Am. Ceram. Soc. 86 [4] (2003) 676685 
[30] K. A. Unocic, B. A. Pint, Oxidation behavior of co-doped NiCrAl alloys in dry and wet air, Surf. Coat. Technol. 237 (2013) 8-15

[31] X. Wang, A. Atkinson, Piezo-spectroscopic mapping of the thermally grown oxide in thermal barrier coatings, Mater. Sci. Eng. A 465 (2007) 49-58

[32] J. Ramírez-Rico, A.R. de Arellano-López, J. Martínez-Fernández, J.I. Pena, A. Larrea, Crystallographic texture in Al2O3-ZrO2 (Y2O3) directionally solidified eutectics, J. Eur. Ceram. Soc. 28 (2008) 2681-2686 
Table I. Nominal compositions of the investigated BC thermal spray powders in wt.\%.

\begin{tabular}{|c|c|c|c|c|c|c|c|}
\cline { 2 - 8 } \multicolumn{1}{c|}{} & Co & Ni & Cr & Al & Y & Hf & Si \\
\hline Co-based & Bal. & 32 & 21 & 8 & 0.5 & - & - \\
\hline Ni-based & 22 & Bal. & 17 & 12 & 0.5 & 0.5 & 0.4 \\
\hline
\end{tabular}


Table II. Summary of the experimental parameters.

\begin{tabular}{|c|c|c|c|}
\hline Experiment & Temperature $\left[{ }^{\circ} \mathbf{C}\right]$ & Environment & EDM hole \\
\hline 1 & 1100 & Air & No \\
\hline 2 & 1050 & Air & No \\
\hline 3 & 1050 & Air $+10 \% \mathrm{H}_{2} \mathrm{O}$ & No \\
\hline 4 & 1000 & Air & Yes \\
\hline 5 & 1000 & Air $+10 \% \mathrm{H}_{2} \mathrm{O}$ & Yes \\
\hline 6 & 950 & Air & Yes \\
\hline
\end{tabular}


Table III. Measured surface roughness parameters and derived interface parameters.

\begin{tabular}{|c|c|c|c|c|}
\hline Surface preparation & $\boldsymbol{S}_{\boldsymbol{a}}[\boldsymbol{\mu \mathrm { m }}]$ & $\boldsymbol{S}_{\boldsymbol{d q}}$ & Amplitude $\boldsymbol{A}[\boldsymbol{\mu m}]$ & Wavelength $\boldsymbol{L}[\boldsymbol{\mu m}]$ \\
\hline 120 grit & 0.67 & 0.131 & 1.65 & 56 \\
\hline 600 grit & 0.19 & 0.057 & 0.47 & 37 \\
\hline
\end{tabular}


Table IV. Coefficients of thermal expansion for the BC materials and $\alpha$-alumina calculated based on temperature-dependent formulas $[25,26]$.

\begin{tabular}{|c|c|c|c|}
\hline Temperature $\left[{ }^{\circ} \mathbf{C}\right]$ & CoNiCrAlY $\left[\mathbf{1 0}^{-\mathbf{6}} \mathbf{K}^{-1}\right]$ & NiCoCrAIY $\left[\mathbf{1 0}^{-\mathbf{6}} \mathbf{K}^{-\mathbf{1}}\right]$ & $\boldsymbol{\alpha}$-alumina $\left[\mathbf{1 0}^{-\mathbf{6}} \mathbf{K}^{-1}\right]$ \\
\hline 20 & 13.22 & 12.84 & 6.65 \\
\hline 120 & 13.32 & 13.2 & 6.89 \\
\hline 220 & 13.69 & 13.64 & 7.11 \\
\hline 320 & 14.21 & 14.11 & 7.3 \\
\hline 420 & 14.77 & 14.6 & 7.48 \\
\hline 520 & 15.35 & 15.1 & 7.66 \\
\hline 620 & 15.93 & 15.59 & 7.83 \\
\hline 720 & 16.51 & 16.09 & 7.99 \\
\hline 820 & 17.1 & 16.59 & 8.15 \\
\hline 920 & 17.69 & 17.08 & 8.3 \\
\hline 1020 & 18.29 & 17.58 & 8.44 \\
\hline 1120 & 18.88 & 18.08 & 8.58 \\
\hline
\end{tabular}


Figure 1. Luminescence spectrum of an oxide scale under stress with characteristic doublepeak structure (R1, R2) and corresponding pseudo-Voigtian fit in comparison to the fit of the reference spectrum obtained for alumina powder.

Figure 2. Measured oxide scale thicknesses determined by image analyses for the two BC compositions and roughness values as a function of exposure time. (W) denotes those specimens exposed in air with 10 vol. $\% \mathrm{H}_{2} \mathrm{O}$.

Figure 3. SEM images of the polished cross sections of a) the 600 grit surface of the CoNiCrAlY sample and b) the 120 grit surface of the NiCoCrAlY sample after 20 cycles at $1100^{\circ} \mathrm{C}$ in air.

Figure 4. Comparison of the measured mean biaxial stresses in the TGO scales to the theoretically obtained average stresses, which were derived from the calculated stress distributions and are displayed by the patterned areas. The calculated stress amounts are proportional to the temperature. Standard errors are given with regard to the experimental results. (W) denotes those specimens exposed in air with 10 vol. $\% \mathrm{H}_{2} \mathrm{O}$.

Figure 5. Mesh structure of the TGO scale and exemplary calculated stress field distribution of a $3 \mu \mathrm{m}$ thick TGO layer on the rougher surface of a Ni-based BC sample after cycling at $1000^{\circ} \mathrm{C}$. 


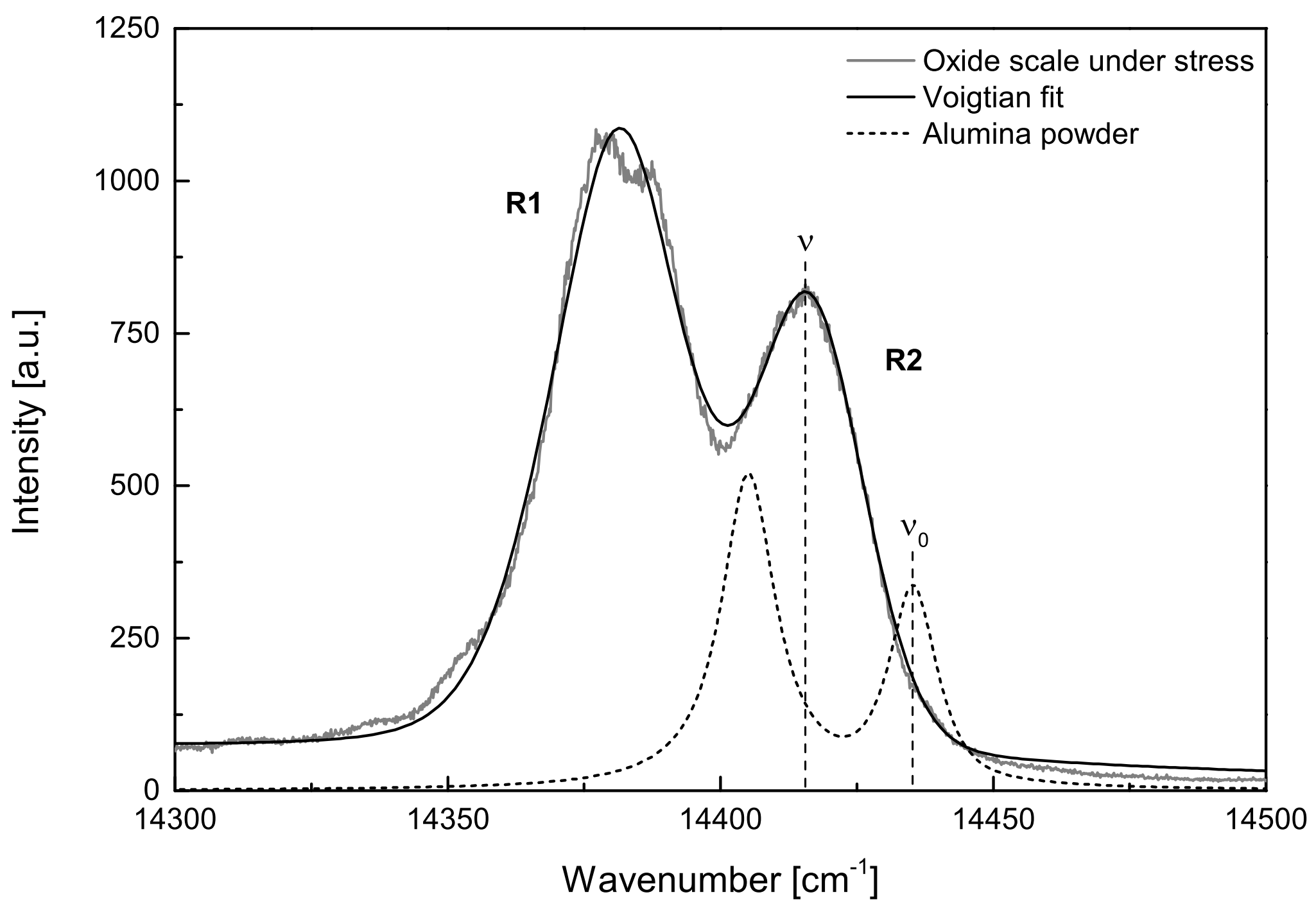



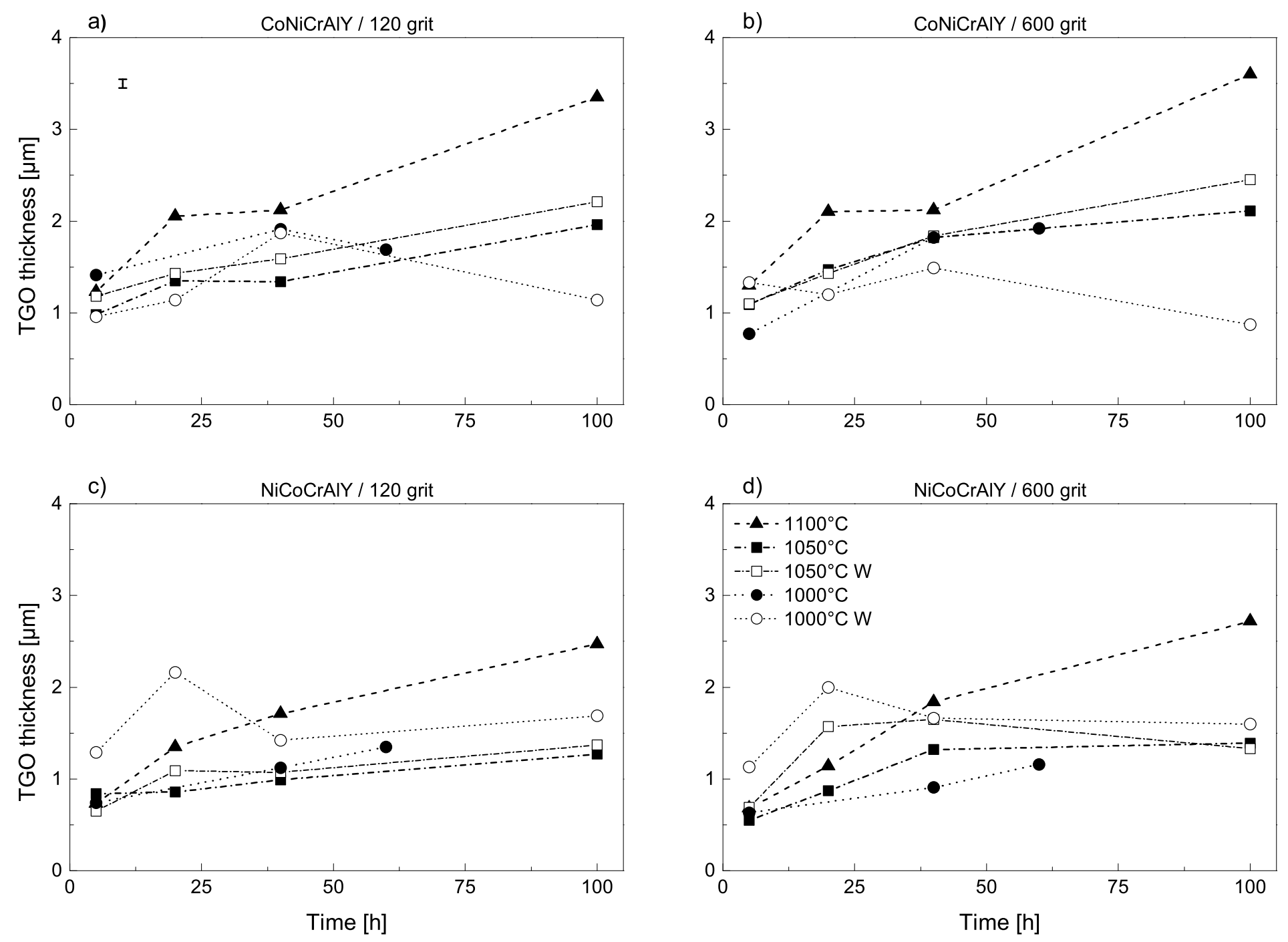


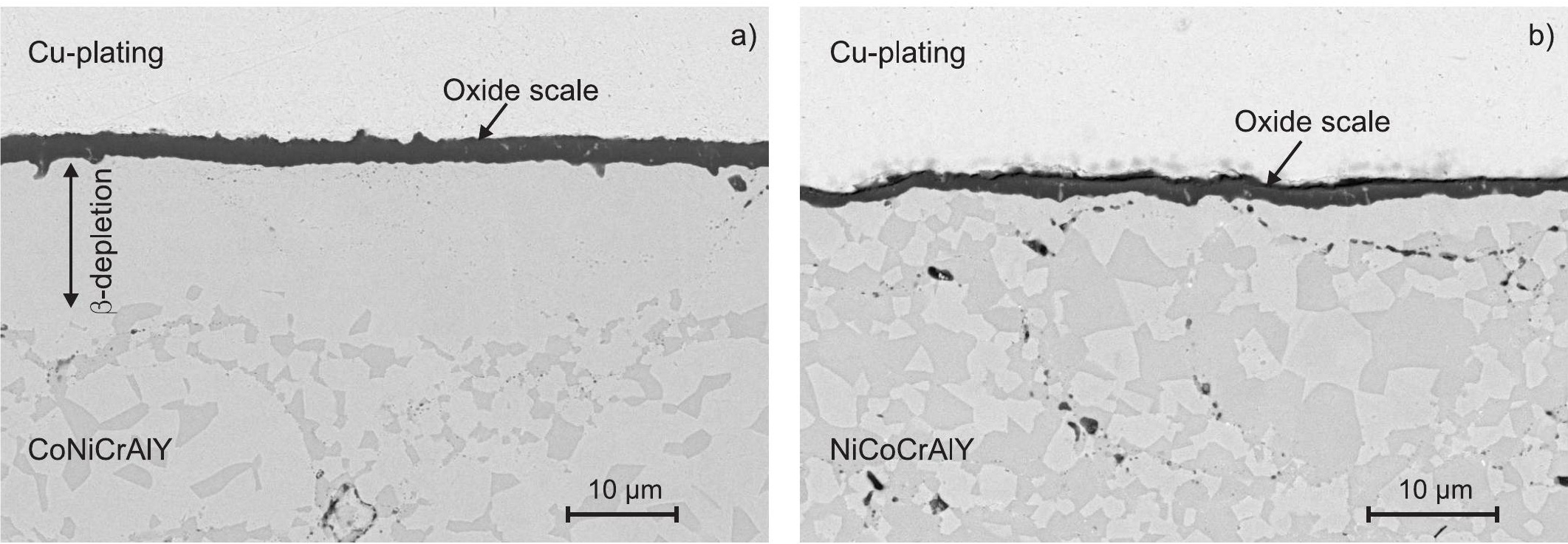



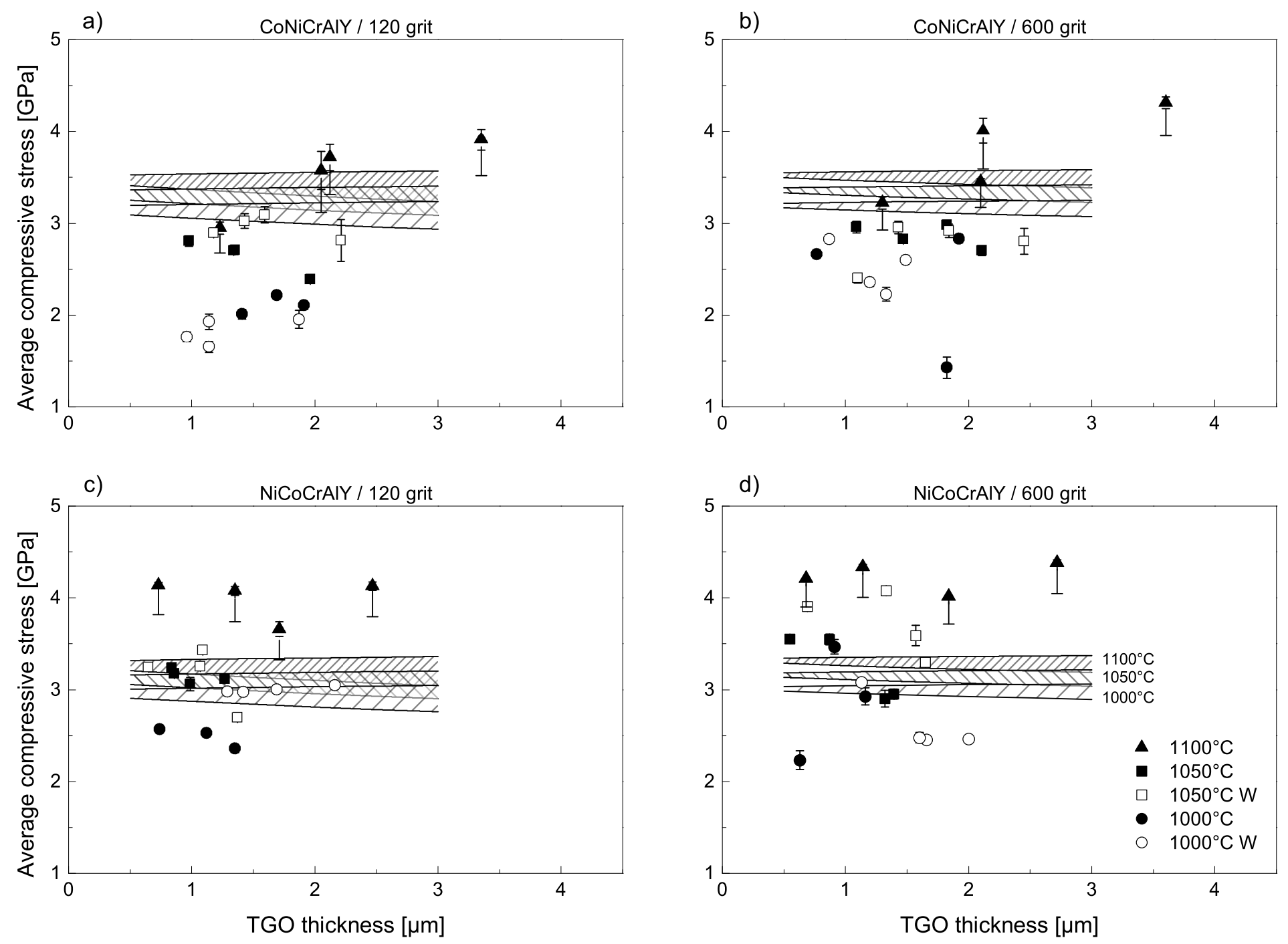


\section{Mesh}

Normal stress ( $x$-direction) [GPa]
$-3.3$
$-3.15$
$-3.0$
$-2.85$
$-2.7$

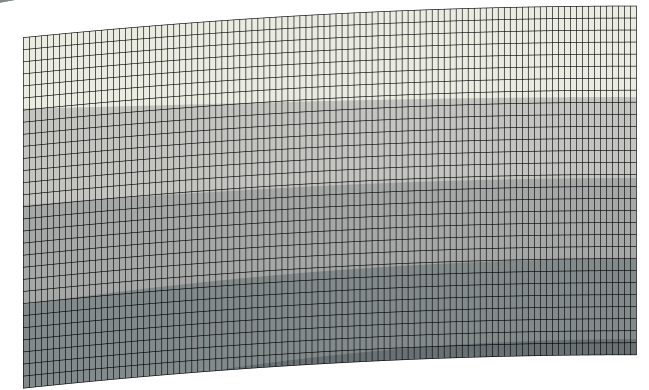

